\title{
快速生长导致转“全鱼”生长激素基因鲤鱼 临界游泳速度的降低
}

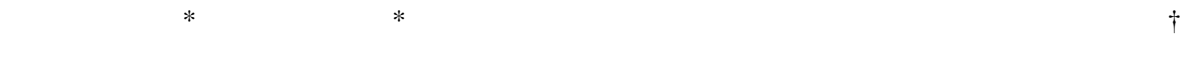 \\ (1) 中国科学院水生生物研究所, 淡水生态与生物技术国家重点实验室, 武汉 430072; (2) 中国科学院研究生院, 北京 100049; \\ (3) 武汉大学基础医学院遗传学系, 武汉 430071. * 同等贡献.† 联系人, E-mail: zyzhu@ihb.ac.cn)
}

\begin{abstract}
摘要 进化生物学研究表明, 快速生长赋予生物的优势与劣势之间存在权衡. 硬骨鱼类的生长率与游 泳能力之间也存在这种权衡. 本研究提出假说认为, 快速生长将导致转基因鱼游泳能力减弱. 比较 $\mathrm{F}_{3}$ 代 转“全鱼”生长激素基因黄河鲤与其对照鱼的生长和临界游泳速度, 表明转基因鱼的平均体重是对照鱼 的 1.4 1.9 倍, 平均特定生长率比对照鱼高出 6\% 10\%, 而其绝对临界游泳速度和相对临界游泳速度的 平均值分别比对照鱼低 $22 \%$ 和 $24 \%$. 本研究暗示, 具有快速生长效应的转基因黄河鲤表现出低劣的游泳 能力, 这也证实了生长率和游泳能力之间存在权衡, 即同一物种的个体生长率越高, 临界游泳速度则越 低.
\end{abstract}

关键词 转基因鱼 生长 游泳能力 权衡 生长激素 临界游泳速度

自 1985 年世界上第一批转基因鱼诞生以来 ${ }^{[1]}$, 快速生长的转基因鱼研究已取得了重大进展. 快速 生长转基因鱼有望在转基因动物中率先实现市场化 [2,3]. 目前, 我国已经建立了稳定遗传的、具有快速生 长效应的转“全鱼”生长激素基因黄河鲤家系, 并且 正在对其生态安全和食品安全性问题进行全面评估 [3,4]. 游泳能力是影响鱼类适合度的重要因素之一 5$]$. 深入了解快速生长对鱼类游泳能力的影响是准确评 估转基因鱼生态安全的重要基础.

大量研究表明, 冷血动物随生长环境调节生长 率使其保持最佳状态, 而不是始终处于最大状态 [6]. 进化生物学方面的研究表明, 快速生长赋予生物的 优势与劣势之间存在权衡 $[5,7,8]$. 硬骨鱼类的生长率 与游泳能力之间也可能存在权衡. Oris等人 [9]首次对 胖头鱥(Pimephales promelas)生长率与游泳能力之间 的关系进行研究, 发现生长率高的个体临界游泳 速度反而低. 类似的结果在虹鳟 (Oncorhynchus mykiss) ${ }^{[10,11]}$ 、大西洋银汉鱼(Menidia menidia $)^{[12,13]}$ 、 三棘刺魚(Gasterosteus aculeatus) ${ }^{[14]}$ 和快速生长转生 长激素基因银大马哈鱼(Oncorhynchus kisutch)中也有 报道.

尽管如此, 关于快速生长转基因鱼游泳速度的
报道却不完全一致. 快速生长转基因银大马哈鱼幼 鱼的临界游泳速度仅有相同大小对照鱼的一半 [15]; 成年转基因银大马哈鱼的临界游泳速度也显著低于 对照鱼, 游泳效率较差 ${ }^{[16]}$; 而快速生长转基因大西 洋鲑(Salmo salar)和罗非鱼(Oreochromis sp.)的游泳 能力与对照鱼之间并没有显著差异 ${ }^{[17,18]}$. 这种结果 的差异或许是由于不同品系或种类的转基因鱼之间 促生长效应的差异所造成的. 进一步对不同品系快 速生长转基因鱼的游泳能力进行研究, 将有助于最 终阐明造成上述差异的主要原因.

临界游泳速度 $\left(U_{\text {crit }}\right)$ 是衡量鱼类游泳能力的一项 重要指标, 其测定方法简而易行 ${ }^{[19,20]}$. 本研究采用测 定临界游泳速度的方法, 来检验快速生长是否会导 致 $\mathrm{F}_{3}$ 代转“全鱼”生长激素基因黄河鲤临界游泳速度 的降低.

\section{1 材料与方法}

(i ) 生长实验. 采用显微注射“全鱼”生长激素 基因 pCAgcGHc 的方法获得 $\mathrm{P}_{0}$ 代转基因鱼. 该“全 鱼”生长激素基因表达载体的重组基因元件来自我国 鲤科养殖鱼类, 包括鲤鱼 $\beta$-actin 基因启动子和草鱼 (Ctenopharyngodon idellus)生长激素基因 cDNA. 实

国家自然科学基金(批准号 : 30400056,30130050)。国家重点基础研究发展计划(批准号:20016B109006)和国家高技术研究发展计划(批准 号: 2004AA213120)资助项目 
验鱼是 2005 年 4 月 14 日由 $\mathrm{F}_{2}$ 代转“全鱼”生长激素 基因黄河鲤雄鱼与对照雌鱼的杂交子代，其中转基 因鱼和对照鱼个体均为 50\%(结果未发表). 实验鱼苗 饲养在武汉多福科技农庄一土池塘中 $($ 长 $\times$ 宽 $\times$ 深 $=$ $33 \mathrm{~m} \times 20 \mathrm{~m} \times 1.0 \mathrm{~m}) .106$ 日龄时, 随机选择转基因 鱼和对照鱼各 500 尾, 分别饲养在两个与上述条件一 致的池塘中. 实验期间, 每天饱食投喂实验鱼, 并在 实验鱼 160, 190, 221 和 251 日龄时, 分 4 次分别随机 测量转基因和对照鱼的体重(数量见表 1). 特定生长 率计算公式为: $\mathrm{SGR}=100 \times\left(\ln W_{t}-\ln W_{0}\right) / t$, 其中 $W_{t}$ 为称重时的体重; $W_{0}$ 为初始体重; $t$ 为生长天数.

(ii) 游泳实验. 2005 年 6 月 15 日, 将 $F_{3}$ 代转 基因黄河鲤从多福科技农庄运回室内循环水系统饲 养. 为了获得与转基因鱼体重相匹配的对照鱼, 来自 河南水产研究所的对照黄河鲤于 3 个月后运回室内 饲养, 饲养条件与转基因鱼一致. 2006 年 1 月 $4 \sim 23$ 日, 对 16 尾体重相似的转基因鱼和对照鱼(各 8 尾) 进行了临界游泳速度的测定. 游泳实验采用 Bretttype 游泳仪进行, 单尾测定. 测试之前, 实验鱼饥饿 12 h (08:30 20:30), 然后轻轻地放入游泳仪中, 启 动游泳仪, 使实验鱼在 $10 \mathrm{~cm} / \mathrm{s}$ 水流中适应过夜. 经 过 $12 \sim 14 \mathrm{~h}$ 的适应, 实验鱼在 $20 \mathrm{~cm} / \mathrm{s}$ 水速中顶水活 动 $10 \mathrm{~min}$, 然后调节游泳舱体中水流使其每 $20 \mathrm{~min}$ 增加 $10 \mathrm{~cm} / \mathrm{s}$, 当实验鱼停在舱体后端电极处不再顶 水游动时, 关闭游泳仪并记录在该速度下顶水游泳 的时间. 待实验鱼在静水中恢复过来之后, 从舱体中 取出, 放入适量的麻醉剂中, 取出并测量其体重、体 长、最大体高和最大体宽(重量精确到 $0.01 \mathrm{~g}$, 长度精 确到 $0.01 \mathrm{~cm})$. 临界游泳速度计算公式为: $U_{\text {crit }}(\mathrm{cm} / \mathrm{s})$ $=u_{\mathrm{i}}+\left(t_{\mathrm{i}} / t_{\mathrm{ii}} \times u_{\mathrm{ii}}\right)$, 其中 $u_{\mathrm{i}}$ 指实验鱼在疲劳之前经历的 倒数第二段的速度; $u_{\mathrm{ii}}$ 指速度增加 $(10 \mathrm{~cm} / \mathrm{s}) ; t_{\mathrm{i}}$ 指实验 鱼在最大水流速度中持续的时间; $t_{\mathrm{ii}}$ 指指定时间段 $(20 \mathrm{~min})$. 相对临界游泳速度 $(\mathrm{BL} / \mathrm{s})=U_{\mathrm{crit}}(\mathrm{cm} / \mathrm{s}) /$
体长. 条件因子 $(\mathrm{CF})=100 \times \mathrm{BW} / \mathrm{BL}^{3}$, 其中 $\mathrm{BW}$ 和 $B L$ 分别指实验鱼的体重和体长.

(iii) 统计方法. 生长实验中不同品系之间的比 较采用 Mann-Whitney $U$ 检验。一元方差分析 (ANOVA)用于不同品系之间温度、体重、体长、全 长和条件因子的比较. 品系间绝对临界游泳速度的 比较, 以温度和体长为协变量, 采用斜方差分析 (ANCOVA). 相对临界游泳速度的比较以温度为协变 量进行协方差分析(ANCOVA). $P<0.05$ 时表示差异 显著.

\section{2 结果}

\section{1 生长实验}

转基因鱼的体重和特定生长率均显著高于对照 鱼(表 1). 不同生长阶段转基因鱼的体重分别是对照 鱼的 1.4 1.9 倍, 其特定生长率分别比对照鱼高出 6\% 10\%.

\section{2 游泳实验}

转基因鱼体重和对照鱼体重相当 $(P>0.68)$, 体 长显著大于对照鱼, 而其条件因子则显著低于对照 鱼 $(P<0.05)$ (表 2). 尽管各实验鱼测定时的水温不同, 但转基因鱼和对照鱼之间平均测定温度没有显著差 异 $(P>0.31)$ (表 2). 转基因鱼的绝对和相对临界游泳 速度均显著低于对照鱼 $(P<0.01)$ (见网络版附录, 表 $3)$, 分别比对照鱼低 $22 \%$ 和 $24 \%$.

\section{3 讨论}

现有研究表明, 转“全鱼”生长激素基因鲤鱼 $\mathrm{F}_{1}$ 代 的平均体重是对照鱼的 1.6 倍 ${ }^{[4] ;} \mathrm{F}_{2}$ 代的平均体重是 对照鱼的 1.8 2.5 倍, 特定生长率比对照鱼高出 $10 \%$ $\sim 13 \%$ (结果末发表). 本研究中, $F_{3}$ 代转基因鲤鱼的平 均体重是对照鱼的 $1.4 \sim 1.9$ 倍, 平均特定生长率比对 照鱼高出 $6 \%$ 10\%. 结果表明, “全鱼”生长激素

表 1 生长实验中不同生长阶段 $\mathrm{F}_{3}$ 代转“全鱼”生长激素基因鱼和对照鱼的体重(g)、特定生长率(\%/天)(平均值士标准误差)

\begin{tabular}{|c|c|c|c|c|c|c|c|}
\hline \multirow{2}{*}{ 生长阶段 } & \multirow{2}{*}{ 变量 } & \multicolumn{4}{|c|}{ 品系 } & \multicolumn{2}{|c|}{ Mann-Whitney $U$ 检验 } \\
\hline & & \multicolumn{2}{|l|}{ 转基因鱼 } & \multicolumn{2}{|l|}{ 对照鱼 } & $Z$ 值 & $P$ 值 \\
\hline \multirow[t]{2}{*}{160 天 } & 体重 & $316.82 \pm 10.22$ & $n=33$ & $223.05 \pm 3.58$ & $n=43$ & 6.58 & $<0.001$ \\
\hline & 特定生长率 & $4.02 \pm 0.02$ & & $3.81 \pm 0.01$ & & 6.58 & $<0.001$ \\
\hline \multirow[t]{2}{*}{190 天 } & 体重 & $396.64 \pm 9.53$ & $n=53$ & $247.07 \pm 9.60$ & $n=27$ & 6.50 & $<0.001$ \\
\hline & 特定生长率 & $3.50 \pm 0.01$ & & $3.26 \pm 0.02$ & & 6.50 & $<0.001$ \\
\hline \multirow[t]{2}{*}{221 天 } & 体重 & $525.64 \pm 13.18$ & $n=45$ & $282.85 \pm 9.54$ & $n=47$ & 7.87 & $<0.001$ \\
\hline & 特定生长率 & $3.14 \pm 0.01$ & & $2.85 \pm 0.01$ & & 7.87 & $<0.001$ \\
\hline \multirow[t]{2}{*}{251 天 } & 体重 & $531.15 \pm 10.63$ & $n=46$ & $307.24 \pm 10.46$ & $n=46$ & 8.07 & $<0.001$ \\
\hline & 特定生长率 & htips:/2.77 \pm 0.01 & & $2.55 \pm 0.01$ & & 8.07 & $<0.001$ \\
\hline
\end{tabular}


表 2 游泳实验中 $\mathrm{F}_{3}$ 代转 “全鱼”生长激素基因鱼和对照鱼的测定温度、体重、体长、全长和条件因子(平均值 \pm 标准误)

\begin{tabular}{|c|c|c|c|c|c|}
\hline 品系 & 温度 $/{ }^{\circ} \mathrm{C}$ & 体重/g & 体长 $/ \mathrm{cm}$ & 全长/cm & 条件因子 $/ \mathrm{g} \cdot \mathrm{cm}^{-3}$ \\
\hline 转基因鱼 $(n=8)$ & $9.85 \pm 0.37$ & $73.19 \pm 1.93$ & $15.11 \pm 0.09$ & $18.75 \pm 0.10$ & $2.12 \pm 0.04$ \\
\hline 对照鱼 $(n=8)$ & $9.30 \pm 0.37$ & $73.77 \pm 0.82$ & $14.84 \pm 0.06$ & $18.1 \pm 0.12$ & $2.26 \pm 0.02$ \\
\hline \multicolumn{6}{|c|}{ 一元方差分析(ANOVA) } \\
\hline$F$ 值 & 1.093 & 0.180 & 4.880 & 8.270 & 9.297 \\
\hline 自由度 & 1,14 & 1,14 & 1,14 & 1,14 & 1,14 \\
\hline$P$ 值 & 0.314 & 0.680 & 0.044 & 0.012 & 0.009 \\
\hline
\end{tabular}

基因的促生长效应可以持续到 $\mathrm{F}_{3}$ 代. 生长激素的这 种促生长效应的持续性在 $\mathrm{F}_{4}$ 代转人生长激素基因鲤 鱼中已有报道 ${ }^{[21]}$. 外源生长激素基因的促生长效应 也已在多种生长激素转基因鱼中得到证实. 例如,

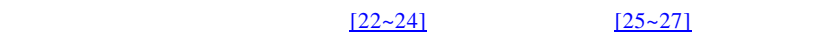
尾撸(Ictalurus punctatus) ${ }^{[28]}$ 、罗非鱼 (Oreochromis niloticus $)^{[29]}$ 、香鱼 (Plecoglossus altivelis $)^{[30]}$ 和野鲮 (Labeo rohita $)^{[31]}$ 等.

本研究中转“全鱼”生长激素基因黄河鲤的平均 绝对临界游泳速度和相对临界游泳速度分别比对照 鱼低 22\%和 24\%, 显示了较弱的游泳能力. 这与在快 速生长转基因鲑鱼中的研究结果一致 [15,16], 也进一 步证实在硬骨鱼类 ${ }^{[9 \sim 13]}$ 和两栖类无尾动物 ${ }^{[5,32,33]}$ 中报 道的生长率和游泳能力之间存在权衡关系, 即快速 生长的个体临界游泳速度较低. 形态差异或许是造 成生长激素转基因鱼游泳能力较弱的因素之一. 研 究表明, 高体、长鯺的鱼类猝发游泳能力强, 而流线 型个体的持续游泳时间较长 [34]. 生长激素转基因显 著地改变了太平洋鲑鱼的尾柄背部、腹部区域和头部 的形态特征 [35]. 快速生长转“全鱼”生长激素基因鲤 鱼躯干肌肉发达, 背部肌肉明显增厚, 在头后部形成 明显隆起 ${ }^{[4]}$. 本研究中, 转基因鱼的体长、全长显著 大于对照鱼, 而条件因子则显著低于对照鱼 $(P<$ 0.05). 综上所述, 快速生长转基因鱼形态特征的改变 以及临界游泳速度的降低使其适合作为模型用于探 讨形态特征和游泳能力之间的关系.

一些研究表明, 个体体细胞的发育妥协于高的 生长速度 ${ }^{[36]}$. 这是由于许多细胞一旦发育成熟就

表 3 游泳实验中 $F_{3}$ 代转“全鱼”生长激素基因鱼和对照鱼 的临界游泳速度(平均值士标准误差)

\begin{tabular}{lcc}
\hline \multicolumn{1}{c}{ 品系 } & $\begin{array}{c}\text { 绝对临界游泳速度 } \\
\left(U_{\text {crit }}, \mathrm{cm} / \mathrm{s}\right)\end{array}$ & $\begin{array}{c}\text { 相对临界游泳速度 } \\
\left(U_{\text {crit }}, \mathrm{BL} / \mathrm{s}\right)\end{array}$ \\
\hline 转基因鱼 $(n=8)$ & $43.58 \pm 1.79$ & $2.89 \pm 0.13$ \\
对照鱼 $(n=8)$ & $53.09 \pm 1.28$ & $3.58 \pm 0.09$ \\
协方差分析(ANCOVA) & & \\
$F$ 值 & 9.575 & 21.974 \\
自由度 & 1,12 & https://engine $1,13 \mathrm{china.com}$ \\
$P$ 值 & 0.009 & $<0.001$ \\
\hline
\end{tabular}

会失去分裂的能力, 从而无法继续维持快速生长 ${ }^{[36]}$. 研究表明, 快速生长的瓜仁太阳鱼(Lepomis gibbosus) 种群头盖骨矿化的速度不及生长速度慢的种群 ${ }^{[37]}$. Arendt等人 ${ }^{[38]}$ 进一步对生长率存在差异的 6 个瓜仁 太阳鱼种群的生长率和鱼鳞抗压强度关系进行研究, 结果表明生长率与鳞片的抗压强度呈负相关关系. 生 长率和鳞片抗压能力之间的权衡也许会影响到鱼类的 其他生活史特征, 包括逃避捕食者的能力和游泳能力. 这或许可以部分地解释转基因银大马哈鱼和 $F_{3}$ 代转基 因黄河鲤生长率和临界游泳速度之间的权衡.

游泳能力影响鱼类的许多生活史特征, 包括捕 食能力、逃避捕食者的能力和生殖洄游 [20]. 因此, 全 面了解转基因鱼的游泳能力对于准确评估其生态安 全具有重要的价值. 已有研究表明, 生长率和游泳能 力之间的生理学权衡通过影响银汉鱼在有捕食压力 情况下的逃避能力, 从而转化为生长率与逃避捕食

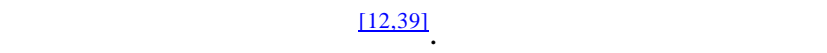
尾撸(I. punctatus)逃避捕食者的能力比对照鱼弱 [40]. 与对照鱼相比, 转基因大西洋鲑(S. salar)和银大马哈 鱼(O. kisutch)也表现出较弱的逃避捕食者的能力 [41 44]. 这意味着快速生长转基因鱼在自然生态系统 中会由于逃避捕食者的能力弱而遭受较高的死亡率. 然而, 进一步的研究有待于验证是由于游泳能力弱 而不是更易于吸引捕食者直接导致了转基因鱼容易 被捕食.

总之, 本研究表明, 快速生长导致 $\mathrm{F}_{3}$ 代转“全鱼” 生长激素基因鲤鱼临界游泳速度的降低.

致谢 感谢解绶启研究员, 雷武、朱晓鸣副研究员, 杨云 霞实验师和聂光汉老师在 Brett-type 游泳仪的设计和组装 过程中给予的大力支持和帮助.

\section{参考文献}

1 Zhu Z, Li G, He L, et al. Novel gene transfer into the fertilized eggs of goldfish(Carassius auratus L. 1758). J Appl Ichthyol, 1985, 1: $31-34$

2 Zbikowska H. Fish can be first-advances in fish transgenesis for commerciab applications.3Transgenic Res, 2003, 12(4): 379389[DOI] 
3 Fu C, Hu W, Wang Y, et al. Developments in transgenic fish in the People's Republic of China. Rev Sci Tech Off Int Epiz, 2005, 24(1): 299-307

4 汪亚平, 胡炜, 吴刚, 等. 转“全鱼”生长激素基因鲤鱼及其 $\mathrm{F}_{1}$ 遗 传分析. 科学通报, 2001, 46(3): 226-229

5 Arendt J D. Reduced burst speed is a cost of rapid growth in anuran tadpoles: Problems of autocorrelation and inferences about growth rates. Funct Ecol, 2003, 17(3): 328-334[DOI]

6 Arendt J D. Adaptive intrinsic growth rates: An integration across taxa. Q Rev Biol, 1997, 72(2): 149-177[DOI]

7 Morgan I J, Metcalfe N B. Deferred costs of compensatory growth after autumnal food shortage in juvenile salmon. Proc R Soc Lond B, 2001, 268(1464): 295-301[DOI]

8 Mangel M, Stamps J. Trade-offs between growth and mortality and the maintenance of individual variation in growth. Evol Ecol Res, 2001, 3(5): 583-593

9 Kolok A S, Oris J T. The relationship between specific growth rate and swimming performance in male fathead minnows (Pimephales promelas). Can J Zool, 1995, 73(11): 2165-2167

10 Gregory T R, Wood C M. Individual variation and interrelationships between swimming performance, growth rate, and feeding in juvenile rainbow trout (Oncorhynchus mykiss). Can J Fish Aquat Sci, 1998, 55(7): 1583-1590[DOI]

11 Gregory T R. Wood C M. Interactions between individual feeding behaviour, growth, and swimming performance in juvenile rainbow trout (Oncorhynchus mykiss) fed different rations. Can J Fish Aquat Sci, 1999, 56(3): 479-486[DOI]

12 Billerbeck J M, Lankford T E, Conover D O. Evolution of intrinsic growth and energy acquisition rates. I . Tradeoffs with swimming performance in Menidia menidia. Evolution, 2001, 55(9): 18631872

13 Munch S B, Conover D O. Nonlinear growth cost in Menidia menidia: Theory and empirical evidence. Evolution, 2004, 58(3): $661-664$

14 Álvarez D, Metcalfe N B. Catch-up growth and swimming performance in threespine sticklebacks (Gasterosteus aculeatus): Seasonal changes in the cost of compensation. Can J Fish Aquat Sci, 2005, 62(9): 2169-2176[DOI]

15 Farrell A P, Bennett W, Devlin R H. Growth-enhanced transgenic salmon can be inferior swimmers. Can J Zool, 1997, 75(2): 335337

16 Lee C G, Devlin R H, Farrell A P. Swimming performance, oxygen consumption and excess post-exercise oxygen consumption in adult transgenic and ocean-ranched coho salmon. J Fish Biol, 2003, 62(4): 753-766[DOI]

17 Stevens E D, Sutterlin A, Cook T. Respiratory metabolism and swimming performance in growth hormone transgenic Atlantic salmon. Can J Fish Aquat Sci, 1998, 55(9): 2028-2035[DOI]

18 McKenzie D J, Martínez R, Morales A, et al. Effects of growth hormone transgensis on metablic rate, exercise performance and hypoxia tolerance in tilapia hybrids. J Fish Biol, 2003, 63(2): 398 -409[DOI]

19 Brett J R. The respiratory metabolism and swimming performance of young sockeye salmon. J Fish Res Bd Can, 1964, 21(5): 11831226

20 Plaut I. Critical swimming speed: Its ecological relevance. Comp Biochem Physiol, 2001, 131A(1): 41-50

21 Fu C, Cui Y, Hung S S O, et al. Growth and feed utilization by $\mathrm{F}_{4}$ human growth hormone transgenic carp fed diets with different protein levels. J Fish Biol, 1998, 53(1): 115-129

22 朱作言, 许克圣, 李国华, 等. 人生长激素基因在泥鳅受精卵显 微注射后的生物学效应. 科学通报, 1986, 31(5): $387-389$

23 朱作言, 许克圣, 谢岳峰, 等. 转基因鱼模型的建立. 中国科学
B 辑, 1989, 2: 147-155

24 崔宗斌, 朱作言, 崔奕波, 等. 转人生长激素基因红鲤 $\mathrm{F}_{2}$ 代阳性 鱼的摄食及代谢研究. 科学通报, 1995, 40(16): 1514-1517

25 Chourrout D, Guyomard R, Houdebine L M. High efficiency gene transfer in rainbow trout (Salmo gairdneri Rich) by microinjection into egg cytoplasm. Aquaculture, 1986, 51(1): 143-150[DOI]

26 Devlin R H, Biagi C A, Yesaki T Y. Growth, viability and genetic characteristics of GH transgenic coho salmon strains. Aquaculture, 2004, 236(1-4): 607-632[DOI]

27 Cook J T, McNiven M A, Richardson G F, et al. Growth rate, body composition/feed digestibility conversion of growth-enhanced transgenic Atlantic salmon (Salmo salar). Aquaculture, 2000, 188(1-2): 15-32[DOI]

28 Dunham R A, Eash J, Askins J, et al. Transfer of metallothionein-human growth hormone fusion gene into channel catfish. Trans Am Fish Soc, 1987, 116(1): 87-91[DOI]

29 Rahman M, Ronyai A, Engidaw B Z, et al. Growth and nutritional trials on transgenic Nile tilapia containing an exogenous piscine growth hormone gene. J Fish Biol, 2001, 59(1): 62-78[DOI]

30 Cheng C, Liu K, Lau E, et al. Growth promotion in Ayu (Plecoglossus altivelis) by gene transfer of the rainbow trout growth hormone gene. Zool Stud, 2002, 41(3): 303-310

31 Venugopal T, Anathy V, Kirankkumar S, et al. Growth enhancement and food conversion efficiency of transgenic fish Labeo rohita. J Exp Zool, 2004, 301A(6): 477-490[DOI]

32 Arendt J, Hoang L. Effect of food level and rearing temperature on burst speed and muscle composition of western spadefoot toad (Spea Hammondii). Funct Ecol, 2005, 19(6): 982-987[DOI]

33 Dayton G H, Saenz D, Baum K A, et al. Body shape, burst speed and escape behavior of larval anurans. Oikos, 2005, 111(3): 582591[DOI]

34 Swain D P. The functional basis of natural-selection for vertebral traits of larvae in the stickleback Gasterosteus aculeatus. Evolution, 1992, 46(4): 987-997[DOI]

35 Ostenfeld T H, McLean E, Devlin R H. Transgenesis changes body and head shape in Pacific salmon. J Fish Biol, 1998, 52(4): 850854[DOI]

36 Starck J M, Ricklefs R E. Avian growth and development. London: Oxford University Press, 1998

37 Arendt J D, Wilson D S. Population differences in the onset of cranial ossification in pumpkinseed (Lepomis gibbosus), a potential cost of rapid growth. Can J Fish Aquat Sci, 2000, 57(2): 351-356

38 Arendt J D, Wilson D S, Stark E. Scale strength as a cost of rapid growth in sunfish. Oikos, 2001, 93(1): 95-100[DOI]

39 Lankford T E, Billerbeck J M, Conover D O. Evolution of intrinsic growth and energy acquisition rates. II. Tradeoffs with vulnerability to predation in Menidia menidia. Evolution, 2001, 55(9): 1873 $-1881$

40 Dunham R A, Chitmanat C, Nichols A. Predator avoidance of transgenic channel catfish containing salmonid growth hormone genes. Mar Biotech, 1999, 1(6): 545-551[DOI]

41 Abrahams M V, Sutterlin A. The foraging and anti-predator behavior of growth-enhanced transgenic Atlantic salmon. Anim Behav, 1999, 58(5): 933-942[DOI]

42 Sundström L F, Devlin R H, Johnsson J I, et al. Vertical position reflects increased feeding motivation in growth hormone transgenic coho salmon (Oncorhynchus kisutch). Ethology, 2003, 109(8): 701-712[DOI]

43 Sundström LF, Lõhmus F, Johnsson J I, et al. Growth hormone transgenic coho salmon pay for growth potential with increased predation mortality. Proc R Soc Lond B, 2004, 271: S350-352

44 Sundström LF, Lõhmus F, Devlin R H. Selection on increased intrinsic growth_rates in coho salmon (Oncorhynchus kisutch). Evolution, 2005, 59(7): $1560-1569$ 
附录 游泳实验中 $\mathrm{F}_{3}$ 代转“全鱼”生长激素基因鱼和对照鱼的测定温度、体重、体长、全长、

条件因子和临界游泳速度

\begin{tabular}{|c|c|c|c|c|c|c|c|}
\hline 品系 & 温度 $/{ }^{\circ} \mathrm{C}$ & 体重/g & 体长/cm & 全长/cm & 条件因子 $/ \mathrm{g} \cdot \mathrm{cm}^{-3}$ & 绝对临界游泳速度 $/ \mathrm{cm} \cdot \mathrm{s}^{-1}$ & 相对临界游泳速度/BL· $\mathrm{s}^{-1}$ \\
\hline \multicolumn{8}{|c|}{ 转基因鱼 } \\
\hline $\mathrm{T}-1$ & 11.2 & 76.40 & 15.0 & 18.7 & 2.26 & 49.33 & 3.29 \\
\hline $\mathrm{T}-2$ & 8.3 & 72.36 & 15.0 & 18.3 & 2.14 & 40.83 & 2.72 \\
\hline $\mathrm{T}-3$ & 8.4 & 75.14 & 15.5 & 19.3 & 2.02 & 46.25 & 2.98 \\
\hline $\mathrm{T}-4$ & 10.0 & 70.93 & 14.7 & 18.2 & 2.23 & 44.64 & 3.04 \\
\hline $\mathrm{T}-5$ & 10.3 & 72.32 & 14.9 & 18.0 & 2.19 & 51.25 & 3.44 \\
\hline T-6 & 9.8 & 69.20 & 15.3 & 19.2 & 1.93 & 39.83 & 2.60 \\
\hline $\mathrm{T}-7$ & 10.9 & 71.00 & 15.0 & 18.9 & 2.10 & 37.33 & 2.49 \\
\hline $\mathrm{T}-8$ & 9.9 & 78.20 & 15.5 & 19.4 & 2.10 & 39.17 & 2.53 \\
\hline \multicolumn{8}{|c|}{ 对照鱼 } \\
\hline $\mathrm{C}-1$ & 8.7 & 73.54 & 15.0 & 18.3 & 2.18 & 52.92 & 3.53 \\
\hline C-2 & 8.7 & 72.05 & 14.8 & 18.3 & 2.22 & 57.25 & 3.87 \\
\hline C-3 & 10.6 & 71.20 & 14.8 & 18.1 & 2.20 & 52.08 & 3.52 \\
\hline C-4 & 10.0 & 70.99 & 14.4 & 17.4 & 2.38 & 49.92 & 3.47 \\
\hline C-5 & 9.3 & 75.43 & 14.9 & 18.1 & 2.28 & 50.83 & 3.41 \\
\hline C-6 & 10.8 & 73.33 & 14.8 & 17.8 & 2.27 & 60.00 & 4.05 \\
\hline C-7 & 8.0 & 76.40 & 15.0 & 18.4 & 2.26 & 50.67 & 3.38 \\
\hline C-8 & 8.3 & 77.00 & 15.0 & 18.4 & 2.28 & 51.08 & 3.41 \\
\hline
\end{tabular}

